\title{
Emergence of carbapenem-resistant Klebsiella spp. infections in a Turkish university hospital: epidemiology and risk factors
}

\author{
Murat Dizbay, Ozlem Guzel Tunccan, Omer Karasahin, Firdevs Aktas \\ Gazi University School of Medicine, Department of Clinical Microbiology and Infectious Diseases, Ankara, Turkey
}

\begin{abstract}
Introduction: Risk factors for nosocomial carbapenem-resistant Klebsiella spp. (CRK) infections were analyzed in this study. Methodology: The incidence, clinical characteristics, risk factors, antimicrobial susceptibility, and outcomes of CRK infections during a seven-year period (2004-2010) were retrospectively analyzed.

Results: A total of 720 patients were included in the study. Carbapenem resistance among Klebsiella spp. was significantly increased between 2003 and $2007(\mathrm{p}<0.001)$. CRK strains were mostly isolated from intensive care units (ICUs) (p < 0.001). Using imipenem and cefoperazone-sulbactam within the prior three months, staying in ICU, receiving immunspressive therapy, receiving $\mathrm{H} 2$ receptor antagonists, having a tracheostomy, using mechanical ventilation, hemodialysis, and having a urinary catheter were found to be significant risk factors for carbapenem-resistant Klebsiella spp. infections. In a multivariate analysis, previously using imipenem (OR 3.35; CI 1.675-6.726, p < 0.001), staying in ICU (OR 3.36; 95\% CI 1.193-9.508; $\mathrm{p}=0.022)$, and receiving H2 receptor antagonist (OR 4.49; 95\% CI 1.011-19.951; $\mathrm{p}=0.048)$ were independently associated with carbapenem resistance. Respiratory tract infections were the most common nosocomial infections. Attack mortality rate was significantly higher in patients infected with CRK strains $(\mathrm{p}<0.001)$. CRK strains showed significantly higher resistance rates to other antibiotics.

Conclusions: The emergence and rapid spread of CRK strains in our hospital is worrisome. The patients in the ICU are at the highest risk for the acquisition of CRK strains. High resistant rates to other antibiotics except colistin and tigecycline limit therapeutic options, and increase mortality rates.
\end{abstract}

Key words: Klebsiella spp.; carbapenem resistance; risk factors; nosocomial infection

J Infect Dev Ctries 2014; 8(1):044-049. doi:10.3855/jidc.3091

(Received 23 October 2012 - Accepted 26 March 2013)

Copyright (C) 2014 Dizbay et al. This is an open-access article distributed under the Creative Commons Attribution License, which permits unrestricted use, distribution, and reproduction in any medium, provided the original work is properly cited.

\section{Introduction}

The increasing prevalence of antimicrobialresistant bacteria has become a serious worldwide problem. Carbapenems have been the drug of choice for treating such severe infections. However, intensive use of these compounds has resulted in the emergence of carbapenem-resistant isolates, leaving few therapeutic options [1]. Carbapenem resistance among Klebsiella spp. has increased in the last decade in many geographical regions of the world, including the USA and Europe. In the USA, the National Healthcare Safety Network (NHSN) reports showed that carbapenem resistance among Klebsiella isolates reached $12 \%$ in invasive device-related infections in 2009-2010 [2]. In Europe, carbapenem-resistant Klebsiella isolates became an important nosocomial pathogen in some countries, particularly in Greece, Italy, and Turkey. Mortality rates of these infections are high because of limited choices of antibiotics. In addition, although there are many reports about hospital outbreaks of carbapenem-resistant Klebsiella spp., only a few of them examine the epidemiological characteristics and risk factors of these infections. Understanding the risk factors of carbapenem-resistant Klebsiella infections will be helpful in preventing these infections. Prolonged hospitalization, staying in the intensive care unit, using invasive devices, immunosupression, and multiple antibiotic agents were reported as risk factors previously [1]. More studies are needed to determine the risk factors for the acquisition of carbapenem-resistant Klebsiella spp. infections in hospital settings.

In this study, we aimed to identify risk factors for nosocomial carbapenem-resistant Klebsiella spp. infections, and to contrast those factors with those for carbapenem-susceptible Klebsiella spp. infections.

\section{Methodology}

Patients who had nosocomial Klebsiella infections between January 2004 and December 2010 were 
included in the study. Nosocomial infection (NI) surveillance was performed prospectively during the period. The data were obtained from infection control committee records. The diagnosis of NI was assessed according to Centers for Diseases Control and Prevention (CDC) criteria [3]. The following data were collected and analyzed from the patients: age, sex, hospital ward, length of hospitalization, prior use of various antibiotics within the last three monts, risk factors and underlying diseases of the patients, type of NI, microbiological data, and outcome. To assay the demographic data, each patient was assessed once. More than one isolate of Klebsiella related to an individual nosocomial infection attack in patients was included in the study. The data about risk factors for carbapenem-resistant Klebsiella spp. infection were given through the number of attacks.

Microorganisms were identified by the BBL Crystal Enteric/Nonfermenter ID Kit (Becton Dickinson, Franklin Lakes, USA). The in vitro activities of antimicrobial agents were tested against the clinical isolates of Klebsiella spp. using the disk diffusion method according to the Clinical and Laboratory Standards Institute (CLSI) criteria [4].

The statistical analysis of the data was performed using the SPSS version 17.0 software package. Categorical variables were analyzed using Chi-square or Fisher's exact test when appropriate. To test the independence of the risk factors for carbapenem resistance, a multivariate analysis was performed by logistic regression. Statistical significance was set at a $\mathrm{p}$ value of $<0.05$.

\section{Results}

Klebsiella spp. was isolated from 840 attacks in 720 patients as a causative agent of nosocomial infection. During the study period, Klebsiella spp. was the etiological agent in $10.1 \%$ of NI isolates, and in $14.9 \%$ of Gram-negative microorganisms hospital wide during the study period. Its incidence and percentage among Gram negatives did not significantly change over the years. Even though the incidence of Klebsiella spp. infection did not change, carbapenem resistance among Klebsiella spp. significantly increased between 2003 and 2007 (p < 0.001 ). The increase in carbapenem resistance was evident between the periods 2004-2008 and 2009-2010 (Table 1).

The male/female ratio was 1.24 ; mean age was 45.40 years ( $\mathrm{SD} \pm 28.68$ years, median 54 years). In total, $41.1 \%$ of Klebsiella spp. infections occurred in intensive care units (ICUs). Carbapenem-resistant strains were more frequent $(78.57 \%)$ than carbapenem-susceptible strains $(39.1 \%)$ in ICUs (p < $0.001)$. Using imipenem and cefoperazone-sulbactam within the prior three months, staying in the ICU, receiving immunspressive therapy, receiving $\mathrm{H} 2$ receptor antagonists, having a tracheostomy, using mechanical ventilation, having hemodialysis, and having a urinary catheter were found to be significant risk factors for carbapenem-resistant Klebsiella spp. infections. Patient characteristics are shown in Table 2. In a multivariate analysis, prior use of imipenem (OR 3.35; CI 1.675-6.726, $\mathrm{p}<0.001$ ), staying in the ICU (OR 3.36; 95\% CI 1.193-9.508; $\mathrm{p}=0.022$ ), and receiving $\mathrm{H} 2$ receptor antagonist (OR 4.49; 95\% CI 1.011-19.951; $\mathrm{p}=0.048)$ were independently associated with carbapenem resistance (Table 2).

The percentage of carbapenem-resistant Klebsiella spp. was significantly higher in respiratory tract infections $(p<0.001)$. There was no statistical difference among other infections related to carbapenem resistance. Of the isolates, $63.1 \%$ were $K$. pneumoniae, $17.9 \%$ were K. oxytoca, and $19 \%$ of them were not typed. Carbapenem resistance was not different among the subtypes of Klebsiella strains.

The attack mortality rate was higher in patients infected with carbapenem-resistant strains (57.1\%) than in those infected with carbapenem-susceptible strains $(27.4 \%)(\mathrm{p}<0.001)$.

Carbapenem-resistant Klebsiella strains showed significantly higher resistance rates to other antibiotics (Table 3). Tigecycline was tested in only 144 strains and the resistance rates to tigecycline were not different between carbapenem-resistant and carbapenem-susceptible strains. Colistin was tested in 100 strains and no resistance was detected.

Table 1. Incidence of Klebsiella spp. infections and isolation percentages by year

\begin{tabular}{lcccccccc}
\hline & $\mathbf{2 0 0 4}$ & $\mathbf{2 0 0 5}$ & $\mathbf{2 0 0 6}$ & $\mathbf{2 0 0 7}$ & $\mathbf{2 0 0 8}$ & $\mathbf{2 0 0 9}$ & $\mathbf{2 0 1 0}$ & Total \\
\hline Number of Klebsiella spp. isolates & 122 & 127 & 145 & 118 & 104 & 100 & 124 & 840 \\
\% of Klebsiella spp. among NI isolates & 11.7 & 10.3 & 9.3 & 8.2 & 7.5 & 8.5 & 9.2 & 10.1 \\
\% of Klebsiella spp. among Gram-negative & 17.1 & 16.8 & 14.4 & 13.1 & 11.8 & 13.5 & 13.8 & 14.9 \\
isolates & 1.6 & 3.1 & 2.7 & 3.3 & 4.8 & 10 & 10.4 & 5 \\
\% of carbapenem resistance & & & & &
\end{tabular}


Table 2. Risk factors of 840 attacks of Klebsiella spp. infections according to carbapenem resistance

\begin{tabular}{|c|c|c|c|c|}
\hline \multirow[b]{2}{*}{ Charasteristic } & \multicolumn{4}{|c|}{ No. (\%) of Klebsiella spp. infections } \\
\hline & CPM R $\mathbf{R}^{\mathrm{a}}$ & CPM S $^{\mathrm{b}}$ & Total & $\mathbf{p}$ \\
\hline Setting, ICU ${ }^{\mathrm{c}}$ & $33(78.57)$ & $312(39.1)$ & $345(41.1)$ & $<0.001$ \\
\hline Age & $52.05 \pm 26.82$ & $45.02 \pm 28.75$ & $45.40 \pm 28.68$ & 0.149 \\
\hline Prior duration of hospitalization & $28.17 \pm 25.20$ & $31.27 \pm 57.86$ & & 0.727 \\
\hline \multicolumn{5}{|l|}{ Underlying diseases / risk factors } \\
\hline Diabetes mellitus & $5(11.1)$ & $111(13.9)$ & $116(13.8)$ & 0.930 \\
\hline Trauma & $2(4.7)$ & $32(4.01)$ & $34(4.04)$ & 0.999 \\
\hline Immunsupressive therapy & $18(42.8)$ & $161(20.1)$ & $279(33.2)$ & 0.002 \\
\hline $\mathrm{H} 2$ antagonist & $40(95.2)$ & $519(65)$ & $559(66.5)$ & $<0.001$ \\
\hline Neutropenia & $2(4.7)$ & $53(6.6)$ & $55(6.54)$ & 0.941 \\
\hline Tracheostomy & $10(23.8)$ & $70(8.7)$ & $80(9.5)$ & 0.008 \\
\hline Urinary catheter & $31(73.8)$ & $430(53.8)$ & $461(54.9)$ & 0.015 \\
\hline Kidney failure & $4(9.5)$ & $44(5.5)$ & $48(5.7)$ & 0.427 \\
\hline Transfusion & $10(23.8)$ & $166(20.8)$ & $176(20.1)$ & 0.61 \\
\hline Endoscopy & $12(28.5)$ & $196(24.5)$ & $208(24.7)$ & 0.669 \\
\hline Drainage catheter & $12(28.5)$ & $244(30.5)$ & $256(30.4)$ & 0.934 \\
\hline Prior use antibiotics & $36(85.7)$ & $572(71.7)$ & $608(72.4)$ & 0.06 \\
\hline Prior use of imipenem & $24(57.1)$ & $147(18.4)$ & $171(20.3)$ & $<0.001$ \\
\hline $\begin{array}{c}\text { Prior use of cefoperazone- } \\
\text { sulbactam }\end{array}$ & $16(38)$ & $110(13.8)$ & $126(15)$ & $<0.001$ \\
\hline \multicolumn{5}{|l|}{ Type of infection } \\
\hline Bloodstream infection & $11(26.1)$ & $189(23.6)$ & $200(22.6)$ & 0.830 \\
\hline Respiratory tract infection & $21(50)$ & $192(24.0)$ & $213(25.35)$ & $<0.001$ \\
\hline
\end{tabular}

${ }^{a}$ CPM R: carbapenem resistant; ${ }^{b}$ CPM S: carbapenem susceptible; ${ }^{\mathrm{c}}$ ICU: intensive care unit

Table 3. Antibiotic resistance rates of Klebsiella spp. according to carbapenem resistance

\begin{tabular}{|c|c|c|c|c|}
\hline \multirow{2}{*}{ Antibiotics } & \multicolumn{4}{|c|}{ No. (\%) of Klebsiella spp infections } \\
\hline & $\mathrm{CPM} \mathrm{R}^{\mathrm{a}}(\mathrm{n}=42)$ & $\mathrm{CPM} \mathrm{S}^{\mathrm{b}}(\mathrm{n}=798)$ & Total $(\mathrm{n}=840)$ & $\mathrm{p}$ \\
\hline Amikacin & $25(59.5)$ & $78(9.7)$ & $103(12.2)$ & $<0.0001$ \\
\hline Amoxicillin clavulanate & $42(100)$ & $601(75.3)$ & $643(80.5)$ & $<0.0001$ \\
\hline Piperacillin-tazobactam & $34(80.9)$ & $281(35.2)$ & $311(37.0)$ & $<0.0001$ \\
\hline Cefotaxime & $40(95.2)$ & $394(49.3)$ & $434(51.6)$ & $<0.0001$ \\
\hline Ceftazidime & $33(78.5)$ & $371(46.4)$ & $404(48.1)$ & $<0.0001$ \\
\hline Cefoperazone-sulbactam & $26(61.9)$ & $253(31.7)$ & $279(33.2)$ & $<0.0001$ \\
\hline Trimethoprim-sulfamethoxazole & $37(88.1)$ & $515(64.5)$ & $552(65.7)$ & 0.001 \\
\hline Cefepime & $36(85.7)$ & $334(41.8)$ & $370(44.0)$ & $<0.0001$ \\
\hline Tigecycline ${ }^{c}$ & $3 / 17(17.6)$ & $17 / 127(13.4)$ & $20 / 144(13.9)$ & 0.861 \\
\hline Colistin $^{\mathrm{c}}$ & $10(100)$ & $60(100)$ & $70(100)$ & \\
\hline
\end{tabular}

${ }^{\mathrm{a}} \mathrm{CPM}$ R: carbapenem resistant; ${ }^{\mathrm{b}} \mathrm{CPM} \mathrm{S}$ : carbapenem susceptible; ${ }^{\mathrm{c}}$ calculated by the number of tested strains 


\section{Discussion}

In recent years, carbapenem resistance in Klebsiella spp. has become a major problem in Turkey as well as the rest of the world. Outbreaks due to carbapenem-resistant Klebsiella spp. have been reported worldwide with increasing frequency $[1,5,6,7]$. In the SENTRY antimicrobial surveillance program (2007-2009), carbapenem resistance was found in $5.3 \%$ of Klebsiella spp., and $3.8 \%$ of the isolates were found to produce a carbapenemase [8].

Carbapenem resistance may occur because of the reduced permeability or carbapenemases. Carbapenemase production is a major resistance mechanism between Enterobacteriaceae. Carbapenemases have been placed in three group of beta-lactamases: class A beta-lactamases such as KPC, class B metallo beta-lactamases such as IMP, VIM, and class D OXA-type beta-lactamases such as OXA48; class D are clinically and epidemiologically the most important enzymes [1]. KPC-type enzymes in carbapenem-resistant $K$. pneumoniae strains were first reported in 2001 in North Carolina [9]. The first outbreak of KPC-producing $K$. pneumoniae outside the USA was in Israel [6]. Almost all European countries are affected by the expansion of carbapenem-resistant Klebsiella spp. In some hospitals in Greece and Italy, carbapenem-resistant Klebsiella spp. has become endemic [7,10].

Although KPC-type carbapenemases are endemic in the countries near Turkey, this enzyme was not described in Turkey until now. However, OXA-type enzymes seem to be primarily a carbapenem resistance mechanism, especially OXA-48, in Turkey. This enzyme was first described in Turkey during an outbreak of K. pneumoniae in Istanbul [11]. OXA-48 producing $K$. pneumoniae was reported from different hospitals in Turkey, as well as in the Middle East, India, and Europe [7,12,13]. In our hospital, the frequency of nosocomial Klebsiella isolates did not change during the study period; however, the frequency of carbapenem-resistant Klebsiella isolates significantly increased. This may be due to an outbreak by the clonal spread of a resistant strain. We found an OXA-48 enzyme in some of the Klebsiella strains, but we could not perform a molecular analysis in all strains. This was a limitation of our study. We focused on analyzing the risk factors of carbapenemresistant Klebsiella spp. infections in our study.

Several risk factors for the acquisition of carbapenem-resistant Klebsiella spp. have been described in the literature. Falagas et al. reported that prior use of quinolones and antipseudomonal penicillins was an independent risk factor associated with the development of carbapenem-resistant $K$. pneumoniae infections [14]. Hussein et al. found an independent association between previous exposure to carbapenems or fluoroquinolones and subsequent development of carbapenem-resistant $K$. pneumoniae (CRKP) infections [5]. Independent risk factors for continued CRKP carriage were antibiotic exposure during the prior three months, receipt of amoxicillinclavulanate, and screening within 90 days of the first culture growing CRKP [15]. Prolonged hospitalization, staying in intensive care unit, using invasive devices, immunosuppression, and multiple antibiotic agents before initial culture were found to be associated with the acquisition of KPC-producing bacteria [1,16]. Our results, similar to other studies, showed that prior use of antibiotics within three months, especially carbapenems and cefoperazone-sulbactams, which are commonly used in our country, was significantly associated with higher carbapenem resistance among nosocomial Klebsiella isolates. Prior use imipenem was found to be an independent risk factor for CRK infections in a multivariate analysis. No significant relationship was detected between other antibiotics and CRK infections.

In our study, staying in the ICU was found to be an independent risk factor for the acquisition of carbapenem-resistant Klebsiella isolates. Serious underlying diseases of the patients, intensive use of invasive devices such as a mechanical ventilator and urinary catheter, use of broad-spectrum antibiotics, and the lack of proper infection control measures may be an explanation for the high incidence of carbapenem resistance in ICUs. Another independent risk factor in the multivariate analysis was the receipt of $\mathrm{H} 2$ receptor antagonist. We did no see this finding in other articles in the literature. Recently, Borer et al. reported from Israel that the presence of a gastric ulcer was associated with the carbapenem-resistant Klebsiella pneumoniae infection [17]. It can be speculated that the use of $\mathrm{H} 2$ receptor antagonists decreases gastric acidity and leads to colonization of carbapenem-resistant Klebsiella spp. in the gut. This is an important mechanism for the acquisition of ventilator-associated pneumonia (VAP) in ICU patients. Respiratory tract infections, including VAP, were the most frequent infections caused by carbapenem-resistant Klebsiella spp. in our study. Tracheostomy, mechanical ventilation, hemodialysis, urinary catheter, and immunspressive therapy, which were found to be significant risk factors in a univarite 
analysis, were similar to the findings of other studies $[14,17,18]$.

Carbapenems are usually the antibiotics of choice for treating serious infections in the ICU. The emergence of carbapenem resistance among Klebsiella spp. limits the therapeutic options for these infections. Resistance rates to other class antibiotics such as penicillins, cephalosporins, quinolones, and aminoglycosides were significantly higher among carbapenem-resistant Klebsiella isolates. In our study, resistance rates to penicillins, cephalosporins, quinolones, and aminoglycosides were found be significantly higher among carbapenem-resistant Klebsiella strains. Colistin resistance was not detected, and suceptibility rates to tigecycline were comparable in both carbapenem-resistant and carbapenemsusceptible Klebsiella isolates. Our results were similar to those of other studies in the literature. Us et al. reported that carbapenem-resistant Klebsiella pneumoniae isolates were resistant to all antimicrobial agents except polymyxins and tigecycline [19]. Won et al. reported that KPC-producing Klebsiella isolates were also resistant to other beta-lactam antibiotics, amikacin, and ciprofloxacin. Colistin and gentamycin were most effective antibiotics in this study [20]. In Italy, Gaibani et al. found that susceptibility to gentamicin, tigecycline, and colistin retained 47 of 52 KPC strains [10]. Patel et al. detected 99 carbapenemresistant Klebsiella isolates between 2004 and 2006. In vitro susceptibility rates were the highest for polymyxin B (90\%) and tigecycline (92\%) [21]. This antimicrobial resistance pattern leads to problems in the treatment of serious infections, especially in the ICU. Tigecycline has limited use in the treatment of respitarory tract, bloodstream, and urinary tract infections because of its pharmacokinetic properties. Therefore, colistin remains an only theraputic option for these infections. Although we did not detect resistance to colistin in our study, some studies showed lower resistance rates to colistin. Colistin resistance rates should be monitored closely.

Limited therapeutic options for these infections may cause an increase in mortality. Several reports have shown that carbapenem resistance was independently associated with an increase in mortality [21-23]. In our study, attack mortality rate was significantly higher in patients infected with carbapenem-resistant strains.

\section{Conclusion}

The emergence and rapid spread of carbapenemresistant Klebsiella spp. in our hospital is worrisome. The patients in the ICU are at the highest risk for acquiring carbapenem-resistant strains. High resistant rates to other antibiotics except colistin and tigecycline limits therapeutic options and increases mortality rates. Limitations of carbapenem overuse, active surveillance, and application of strict infection control measures are necessary for combating these infections.

\section{References}

1. Nordmann P, Cuzon G, Naas T (2009) The real threat of Klebsiella pneumoniae carbapenemase-producing bacteria. Lancet Infect Dis 9: 228-236.

2. Sievert DM, Ricks P, Edwards JR, Schneider A, Patel J, Srinivasan A, Kallen A, Limbago B, Fridkin S (2013) National Healthcare Safety Network (NHSN) Team and Participating NHSN Facilities. Antimicrobial-resistant pathogens associated with healthcare-associated infections: summary of data reported to the National Healthcare Safety Network at the Centers for Disease Control and Prevention, 2009-2010. Infect Control Hosp Epidemiol 34: 1-14.

3. Horan TC, Andrus M, Dudeck MA (2008) CDC/NHSN surveillance definition of health care-associated infection and criteria for specific types of infections in the acute care setting. Am J Infect Control 36: 309-332.

4. Clinical and Laboratory Standards Institute (2005) Performance Standarts for Antimicrobial Susceptibility Testing; 15th Informational Supplement, M100-S15. CLSI: Wayne PA.

5. Hussein K, Sprecher H, Mashiach T, Oren I, Kassis I, Finkelstein R (2009) Carbapenem resistance among Klebsiella pneumoniae isolates: risk factors, molecular characteristics, and susceptibility patterns. Infect Control Hosp Epidemiol 30: 666-671.

6. Leavitt A, Navon-Venezia S, Chmelnitsky I, Schwaber MJ, Carmeli Y (2007) Emergence of KPC-2 and KPC-3 in carbapenem-resistant Klebsiella pneumoniae strains in an Israeli hospital. Antimicrob Agents Chemother 51: 30263029.

7. Grundmann H, Livermore DM, Giske CG, Canton R, Rossolini GM, Campos J, Vatopoulos A, Gniadkowski M, Toth A, Pfeifer Y, Jarlier V, Carmeli Y; CNSE Working Group (2010) Carbapenem-non-susceptible Enterobacteriaceae in Europe: conclusions from a meeting of national experts. Euro Surveill 15. pii: 19711.

8. Castanheira M, Mendes RE, Woosley LN, Jones RN (2011) Trends in carbapenemase-producing Escherichia coli and Klebsiella spp. from Europe and the Americas: report from the SENTRY antimicrobial surveillance programme (200709). J Antimicrob Chemother 66: 1409-1411.

9. Yigit H, Queenan AM, Anderson GJ, Domenech-Sanchez A, Biddle JW, Steward CD, Alberti S, Bush K, Tenover FC (2001) Novel carbapenem-hydrolyzing beta-lactamase, KPC1, from a carbapenem-resistant strain of Klebsiella pneumoniae. Antimicrob Agents Chemother 45: 1151-1161. 
10. Gaibani P, Ambretti S, Berlingeri A, Gelsomino F, Bielli A, Landini MP, Sambri V (2011) Rapid increase of carbapenemase-producing Klebsiella pneumoniae strains in a large Italian hospital: surveillance period 1 March - 30 September2010. Euro Surveill 16. pii: 19800.

11. Carrër A, Poirel L, Eraksoy H, Cagatay AA, Badur S, Nordmann P (2008) Spread of OXA-48-positive carbapenemresistant Klebsiella pneumoniae isolates in Istanbul, Turkey. Antimicrob Agents Chemother 52: 2950-2954.

12. Aktaş Z, Kayacan CB, Schneider I, Can B, Midilli K, Bauernfeind A (2008) Carbapenem-hydrolyzing oxacillinase, OXA-48, persists in Klebsiella pneumoniae in Istanbul, Turkey. Chemotherapy 54: 101-106.

13. Gülmez D, Woodford N, Palepou MF, Mushtaq S, Metan G, Yakupogullari Y, Kocagoz S, Uzun O, Hascelik G, Livermore DM (2008) Carbapenem-resistant Escherichia coli and Klebsiella pneumoniae isolates from Turkey with OXA-48like carbapenemases and outer membrane protein loss. Int $\mathrm{J}$ Antimicrob Agents 31: 523-526.

14. Falagas ME, Rafailidis PI, Kofteridis D, Virtzili S, Chelvatzoglou FC, Papaioannou V, Maraki S, Samonis G, Michalopoulos A (2007) Risk factors of carbapenem-resistant Klebsiella pneumoniae infections: a matched case control study. J Antimicrob Chemother 60: 1124-1130.

15. Ben-David D, Masarwa S, Navon-Venezia S, Mishali H, Fridental I, Rubinovitch B, Smollan G, Carmeli Y, Schwaber $\mathrm{MJ}$; Israel PACF CRKP (Post-Acute-Care Facility Carbapenem-Resistant Klebsiella pneumoniae) Working Group (2011) Carbapenem-Resistant Klebsiella pneumoniae in Post-Acute-Care Facilities in Israel. Infect Control Hosp Epidemiol 32: 845-853.

16. Schwaber MJ, Klarfeld-Lidji S, Navon-Venezia S, Schwartz D, Leavitt A, Carmeli Y (2008) Predictors of carbapenemresistant Klebsiella pneumoniae acquisition among hospitalized adults and effect of acquisition on mortality. Antimicrob Agents Chemother 52: 1028-1033.

17. Borer A, Saidel-Odes L, Eskira S, Nativ R, Riesenberg K, Livshiz-Riven I, Schlaeffer F, Sherf M, Peled N (2011) Risk factors for developing clinical infection with carbapenemresistant Klebsiella pneumoniae in hospital patients initially only colonized with carbapenem-resistant $K$ pneumoniae. Am J Infect Control $40: 421-425$.

18. Gasink LB, Edelstein PH, Lautenbach E, Synnestvedt M, Fishman NO (2009) Risk factors and clinical impact of Klebsiella pneumoniae carbapenemase-producing $K$. pneumoniae. Infect Control Hosp Epidemiol 30: 1180-1185.

19. Us E, Tekeli A, Arikan Akan O, Dolapci I, Sahin F, Karahan ZC (2010) Molecular epidemiology of carbapenem-resistant Klebsiella pneumoniae strains isolated between 2004-2007 in Ankara University Hospital, Turkey. Mikrobiyol Bul 44: 110.

20. Won SY, Munoz-Price LS, Lolans K, Hota B, Weinstein RA, Hayden MK; Centers for Disease Control and Prevention Epicenter Program (2011) Centers for Disease Control and Prevention Epicenter Program. Emergence and rapid regional spread of Klebsiella pneumoniae carbapenemase-producing Enterobacteriaceae. Clin Infect Dis 53: 532-540.

21. Patel G, Huprikar S, Factor SH, Jenkins SG, Calfee DP (2008) Outcomes of carbapenem-resistant Klebsiella pneumoniae infection and the impact of antimicrobial and adjunctive therapies. Infect Control Hosp Epidemiol 29: 1099-2106.

22. Ben-David D, Kordevani R, Keller N, Tal I, Marzel A, GalMor O, Maor Y, Rahav G (2012) Outcome of carbapenem resistant Klebsiella pneumoniae bloodstream infections. Clin Microbiol Infect 18: 54-60.

23. Gupta N, Limbago BM, Patel JB, Kallen AJ (2011)Carbapenem-resistant Enterobacteriaceae: epidemiology and prevention. Clin Infect Dis 53: 60-67.

\section{Corresponding author}

Murat Dizbay

Gazi University School of Medicine, Department of Clinical

Microbiology and Infectious Diseases

Besevler, 06510, Ankara, Turkey

Phone: +90 3122025432

Fax: +903122136333

Email: muratdizbay@gazi.edu.tr

Conflict of interests: No conflict of interests is declared. 